\title{
Bucheinbände und Rezeption der Antikriegsromane von Alexander Moritz Frey und Adrienne Thomas
}

\section{Ina Ulrike Paul}

Freie Universität Berlin

\section{Abstract}

Die autobiographisch unterlegten Antikriegsromane von Alexander Moritz Frey (1881-1957) Die Pflasterkästen. Ein Feldsanitätsroman (1929) und Adrienne Thomas Die Katrin wird Soldat. Ein Roman aus Elsass-Lothringen (1930) literarisieren den Erfahrungsraum des Sanitätsdienstes und der Kriegskrankenpflege; sie kamen in der Weimarer Republik fast gleichzeitig mit Erich Maria Remarques Bestseller Im Westen nichts Neues auf den Buchmarkt - und mussten diesem dank ihrer Qualität zunächst nicht weichen. Ihre Schutzumschläge und Bucheinbände, oft von renommierten Graphiker*innen geschaffen, kommentieren die in ihnen enthaltene Erzählung und sie illustrieren das Vergessen und Erinnern der Werke, die sie umschlossen.

Les deux romans pacifistes à caractère autobiographique, celui d'Alexander Moritz Frey (1881-1957), Die Pflasterkästen. Ein Feldsanitätsroman, de 1929 (Les caisses de pansements. Un roman de service de santé militaire en campagne) et celui d'Adrienne Thomas (1887-1980), Die Katrin wird Soldat. Ein Roman aus Elsass-Lothringen, de 1930 (Catherine soldat, 1933 pour la traduction française), analysent par le moyen de l'écriture l'expérience du service médical de campagne et des soins de santé en temps de guerre ; ils sont sortis sur le marché du livre pendant la République de Weimar, presque en même temps que le best-seller À l'Ouest rien de nouveau d'Erich Maria Remarque auquel, grâce à leur qualité, ils n'ont pas eu dans un premier temps à céder le pas. Les diverses jaquettes et couvertures de ces ouvrages, souvent créées par des graphistes de renom, exposent le récit consigné et illustrent l'oubli et la renaissance qu'ont connus les œuvres qu'ils contiennent.

Two autobiographically inspired anti-war novels, Die Pflasterkästen. Ein Feldsanitätsroman (1929, tr. as The Cross Bearers, 1930) by Alexander Moritz Frey (1881-1957) and Die Katrin wird Soldat. Ein Roman aus Elsass-Lothringen (1930, tr. by Margaret L. Goldsmith as Catherine Joins Up / Katrin Becomes a Soldier, London / Boston, 1931) by Adrienne Thomas make literary capital out of the experiences of wartime medicine and nursing. Published in the Weimar Republic soon after Erich Maria Remarque's All Quiet on the Western Front, they were good enough to hold their own against this bestseller for a time. Their dust jackets and covers, often created by prestigious designers, illustrated the oblivion and memory of the works within and commented on the stories they contained.

\section{Keywords}

Antikriegsromane • Frey (Alexander Moritz) • Thomas (Adrienne) • Schutzumschlag/Bucheinband • Rezeptionsgeschichte

roman anti-guerre $•$ Frey (Alexander Moritz) • Thomas (Adrienne) • reliure /couverture de livre $\bullet$ histoire de la réception

anti-war novels • Frey (Alexander Moritz) • Thomas (Adrienne) • book jacket/ book cover $\bullet$ reception history

Vielleicht hoffen wir es Alle: Dass die Feder mächtiger ist als das Schwert. Dass Zensur den Erfolg eines Buches nicht verhindern kann. Dass verbannte Autor*innen und verbrannte Bücher nicht in Vergessenheit geraten. Und doch: Die nationalsozialistische Verfolgung von Schriftsteller*innen führte ebenso wie die Verbrennung ihrer Bücher vielfach zu einer damnatio memoriae. Karrieren wurden abgebrochen, manche für immer beendet, wie es die Schicksale zweier deutschsprachiger Romanautor*innen der Zwischenkriegszeit erweisen: Wer spricht heute in Deutschland von Alexander Moritz Frey? Wer kennt in Frankreich noch Adrienne Thomas? Beide waren um 1930 erfolgreiche Schriftsteller*innen, deren vergessene pazifistische Romane über den Ersten Weltkrieg erst in den 1980er Jahren neu aufgelegt wurden - etwa in der Reihe Verboten und verbrannt/Exil des Fischer Taschenbuch Verlages oder in den regional spezialisierten Éditions serpenoise.

Man sieht es ihren Büchern an - den Erstausgaben, den späteren Auflagen -, welche Schicksale diese beiden Schriftstellerpersönlichkeiten hatten und wie ihre Werke rezipiert wurden. Die Bucheinbände kommentieren die in ihnen enthaltene Erzählung und sie illustrieren das Vergessen und Erinnern der Werke, die sie umschlossen; der eine oder andere von ihnen berichtet sogar von den Autor*innen - einer Frau, die wie ihr literarisches alter ego als 17jährige Rote 
Kreuz-Helferin den Ersten Weltkrieg an einer ,Erfrischungsstation' im Bahnhof Metz erlebte, und einem Mann, der doppelt so alt war wie sie und als Feldsanitäter Dienst an der ,Westfront' tat. Beider autobiographisch unterlegte Antikriegsromane literarisierten den Erfahrungsraum des Sanitätsdienstes und der Kriegskrankenpflege; sie kamen in der Weimarer Republik gleichzeitig mit Erich Maria Remarques Bestseller Im Westen nichts Neues auf den Buchmarkt - und mussten diesem dank ihrer Qualität anfangs nicht weichen. Schließlich sprechen die Bucheinbände auch von Versuchen nach dem Zweiten Weltkrieg, die einst viel gelesenen Romane zweier Emigrant*innen wieder ins Licht der Öffentlichkeit zu rücken (Sas 2011).

\section{ALEXANDER MORITZ FREY UND SEIN FELD- SANITÄTSROMAN}

Wer kann einen „Feldsanitätsroman“ schreiben? Alexander Moritz Frey (1881-1957) als ehemaliger Sanitäter im Ersten Weltkrieg konnte es. ${ }^{1}$ Der 34jährige Frey hatte in der avantgardistischen Münchener Künstlerszene einen Namen als Autor phantastisch-bizarrer Novellen, als er sich im September 1915 freiwillig zum Kriegsdienst meldete. Er zog nicht aus Kriegsbegeisterung in diesen Krieg; vielmehr hatte er 1914/15 zwei pazifistische Novellen verfasst, die bis 1918 von der Militärzensur unterdrückt wurden (Hoffmann-Walbeck 1984: 82). Als Sanitäter gehörte er dem bayerischen „Regiment List“ an, das während des gesamten Krieges an der ,Westfront' zwischen Verdun und der Somme lag. ${ }^{2}$ Der Schriftsteller-Feldsanitäter verrichtete in den Worten des befreundeten Journalisten Max Kolmsperger (18901966) „trotz seines unsagbaren Abscheus vor Krieg und Kriegsgeschrei im Getöse der Materialschlachten mit einer Hingabe ohne gleichen [...] Samariterdienste."3 Frey selbst blieb bis auf „ein paar Kratzer“ unverletzt: „Links und rechts fielen sie, tot und zerfetzt waren sie, verröchelt sind sie mit dem Kopf in meinem Schoß ... - und ich bin selber dageblieben in dieser sinnlosen, dummen, brutalen Welt" (Frey 1939? zit. nach Ernsting 2007, 16). Die Begegnung mit einem der Meldegänger im Regimentsstab war für Frey schicksalhaft, traf er den Gefreiten Adolf Hitler doch nach dem Krieg wieder. Unmittelbar nach seiner Rückkehr nach München begann Frey mit der literarischen Verarbeitung seiner Kriegserlebnisse - aus seelischer Not und aus finanzieller, denn das in Kriegsanleihen angelegte Familienvermögen war verloren. ${ }^{4}$ Eine kleine, im Juni 1927 in der Weltbühne publizierte Kurzgeschichte Die Maus und die Granate über die Tierquälerei der von Ungeziefer gequälten Soldaten wurde mit verändertem Erzählmodus zum neunten

\footnotetext{
Ernsting 2007; Atze 2006; Asendorf / von Bockel 2006; Walter 1988; HoffmannWalbeck 1984.

2 Frey gehörte der 8. Kompanie des königlich bayerischen Reserve-Infanterieregiments Nr. 16 an, das nach dem im Oktober 1914 gefallenen Kommandeur Oberst Julius List de Ehrennamen "List" erhielt (Weber 2011).

3 Max Kolmsperger, Gedenkrede am 28. Januar 1957 im Bayerischen Rundfunk, zit. nach Greiner-Mai 1984, 250

4 Als erste der damals verfassten Novellen beklagte Die Ernte (1919) die zahllosen Toten als Ernte des Ersten Weltkrieges. Frey nahm sie unter dem neuen Titel Verzweiflung in den
} Band Spuk des Alltags (1920) auf.
Kapitel seines größten Romanerfolges Die Pflasterkästen. Ein Feldsanitätsroman, der 1929 im Gustav Kiepenheuer Verlag in Berlin in einer Erstauflage von 5.000 Exemplaren erschien (Kiepenheuer 1951: 83-85; Thema - Stil - Gestalt 1984).

Hauptfigur des Romans ist Freys literarisches alter ego, der Sanitäter, Schriftsteller und Pazifist Christian Friedrich Funk. Der Roman beschreibt den Kriegsalltag der einfachen Soldaten, ihre Kameradschaft und ihre Verrohung, die Allgegenwart von grauenhafter Verletzung und mörderischem Tod im modernen „Maschinenkrieg“; er kritisiert unfähige und schikanöse Vorgesetzte, die Sinnlosigkeit und Unmenschlichkeit jeden Krieges und die Bedrückungen der leidenden Zivilbevölkerung im umkämpften und misshandelten Frankreich. Die Erzählung schließt mit einer radikalen Absage Funks an den Krieg: „Ich will, will, will die Wahrheit sagen - ich will sagen: Militär und Krieg sind die albernste, schamloseste, dümmste Gemeinheit von der Welt“ (Frey 1984: 245).

Umschlag und Einband der Erstausgabe hatte der Buchgestalter, Illustrator und Bühnenbildner Georg Salter (18971967) entworfen (Fischer 2021, p. 226-232; Schauer 2003: 129-132; Dorén 2003: 133-139; Hansen 2005, mit vorzüglichen Abbildungen). Innovativ fasste er in den 1920er Jahren das Äußere eines Buches als „eigenständiges Kommunikationsmedium" auf und entwarf deshalb „für jedes Buch einen individuell gestalteten Umschlag, der Inhalt und Atmosphäre ebenso berücksichtigte wie die Werbewirkung" (Chrambach 2005). ${ }^{5}$ Heute ist es kaum möglich, auf dem antiquarischen Buchmarkt die Erstauflage der Pflasterkästen im Schutzumschlag zu finden, der ein rot schraffiertes, als Guilloche auf das klassische „Rote Kreuz“ aufgedrucktes Kreuz zeigt. Die feldgraue Schablonenschrift von Autornamen und Titel weckte ein Jahrzehnt nach dem Ende des Weltkriegs bei der Leserschaft noch einschlägige Erinnerungen. ${ }^{6}$ (Abb. 1). Der Umschlag wie der Leineneinband mit roter Beschriftung und olivfarbenem Seitenschnitt entsprachen dem Gestaltungsideal der neuen Sachlichkeit als politischer Kunst.

Auch international erfuhren die Pflasterkästen Anerkennung (Weber 2011: 179). Schon 1929 wurde der Roman als Funk ziekendrager aan het Westelijk front (Sanitäter Funk an der Westfront) ins Niederländische übersetzt, wobei der kulturell adaptierte Titel sowohl die Hauptfigur als auch den Schauplatz beim Namen nannte. ${ }^{7}$ Die Gestaltung mit verschiedenen Schrift- und graphischen Elementen gehört ebenfalls zur neuen Sachlichkeit. (Abb. 2). Nach zwei englischsprachigen Übersetzungen in den USA (1930) und in Großbritannien (1931) unter dem Titel The Cross Bearers (Die Kreuzträger) ${ }^{8}$

5 Hansen (2005: 179) gibt an, dass Salter Bucheinband und Bindung der Pflasterkästen gestaltet habe.

6 Abgewandelt zit. nach Thema - Stil - Gestalt 1984: 468. Umschlagbild in Farbe auf S. 46 (Holstein), Beschreibung dazu S. 26, Rückenansicht des Buches ohne Umschlag S. 44.

7 Der Übersetzer J. L. van der Moer konnte außer im Worldcat mit dem von inm übersetzten Titel http://worldcat.org.ezproxy.uindy.edu/identities/viaf-284481594/ und in der $\mathrm{dbnl}$ (digitale bibliotheek for de Nederlandse letteren) https://www.dbnl.org/auteurs/auteur. php?id=moer050 [Zugriff: 02.07.2020] nicht ausfindig gemacht werden.

8 Die amerikanische, in New York bei Viking Press erschienene Ausgabe trug den irreführenden Untertitel $A$ Story of the Medical Corps, während die britische Übersetzung im Londoner Verlag G. P. Putnam's Sons auf einen Untertitel verzichtete. Die britische Ausgabe zitiert Brian Murdoch (2017), der keine Angaben zum Ubersetzer geben kann („no translator given“) und der Freys Roman „still unjustly neglected“ findet (ebd. 165). - Beide englischsprachigen Ausgaben waren wegen der Corona-Pandemie nicht über internationale Fernleihe zu erhalten. 


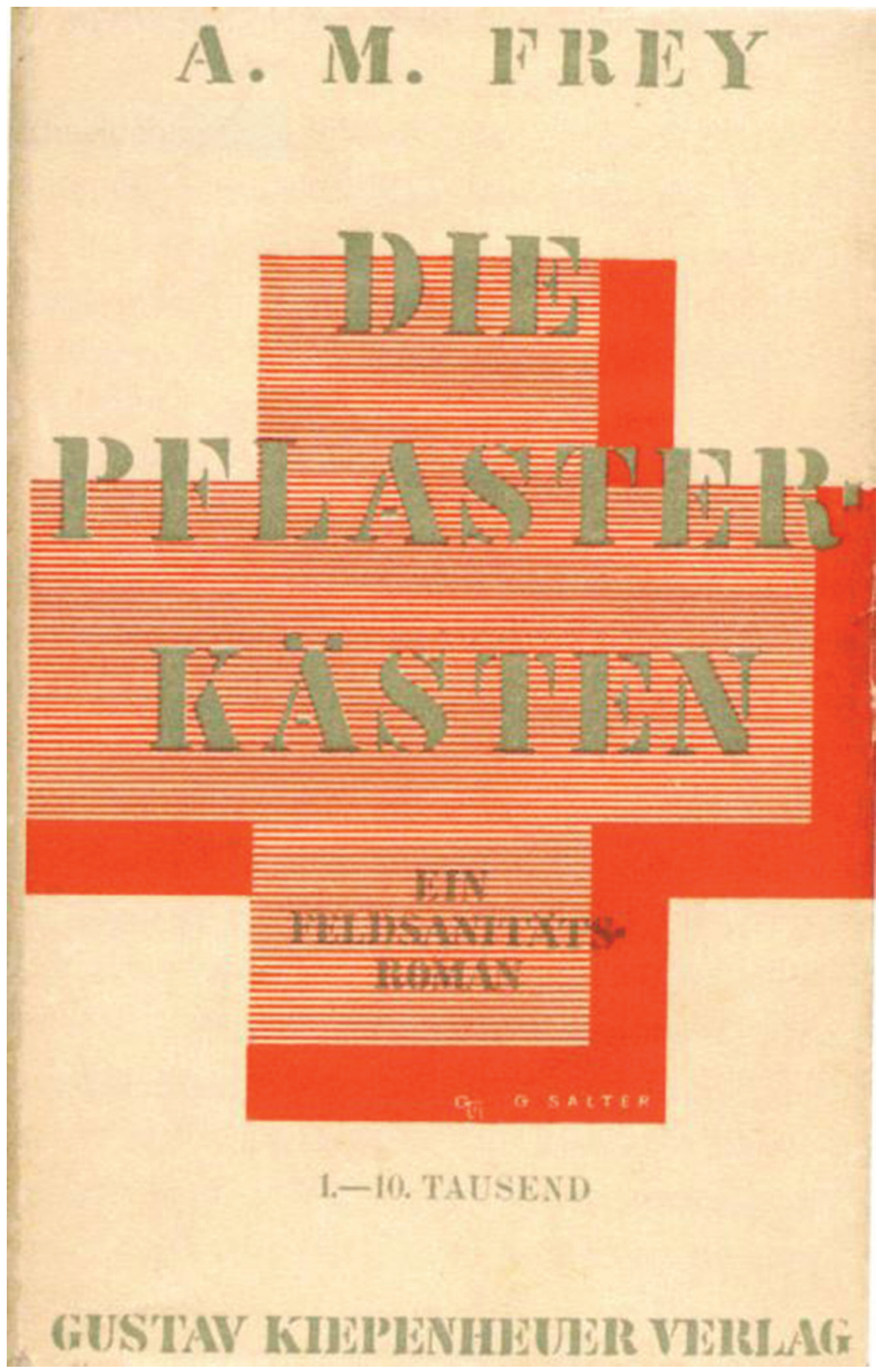

Abb.1:

kam Freys Roman 1931 in Warschau mit dem an das Original anklingenden Titel Apteczka polowa. Powieść (Der FeldVerbandskasten. Ein Roman) auf den Buchmarkt (Ernsting 2007: 91; Frey: 1931). Der Literat und vielsprachige Übersetzer Marceli Tarnowski (1899-1944) verantwortete die polnische Ausgabe. ${ }^{9}$ Der etwa im Vergleich mit der deutschen Erstausgabe expressivere Buchumschlag der polnischen

9 Zu dem polnischen Literaturübersetzer Marceli Tarnowski s. https://pl.wikipedia.org/ (Zugriff: 02.07.2020]. Für Ubersetzungen de
Ausgabe entspricht dem harten Ton und dem bewusst schockierenden ,Stoff' von Freys Pflasterkästen und geht damit über die bloße Sachlichkeit der ,deutschen“ Buchumschläge hinaus: Auf ein nach links kippendes Rotes Kreuz, das den Großteil des schwarzweißen Umschlags einnimmt, ist das zeitgenössische Foto eines Sanitäters montiert, der sich um einen Verwundeten kümmert, wobei hinter ihm ein echter „Pflasterkasten“ zu sehen ist. Das Feld unter dem rechten Arm des Roten Kreuzes füllt die ernste Miene eines ,gueule cassé - eines im Gesicht schwer Verletzten. In der oberen 


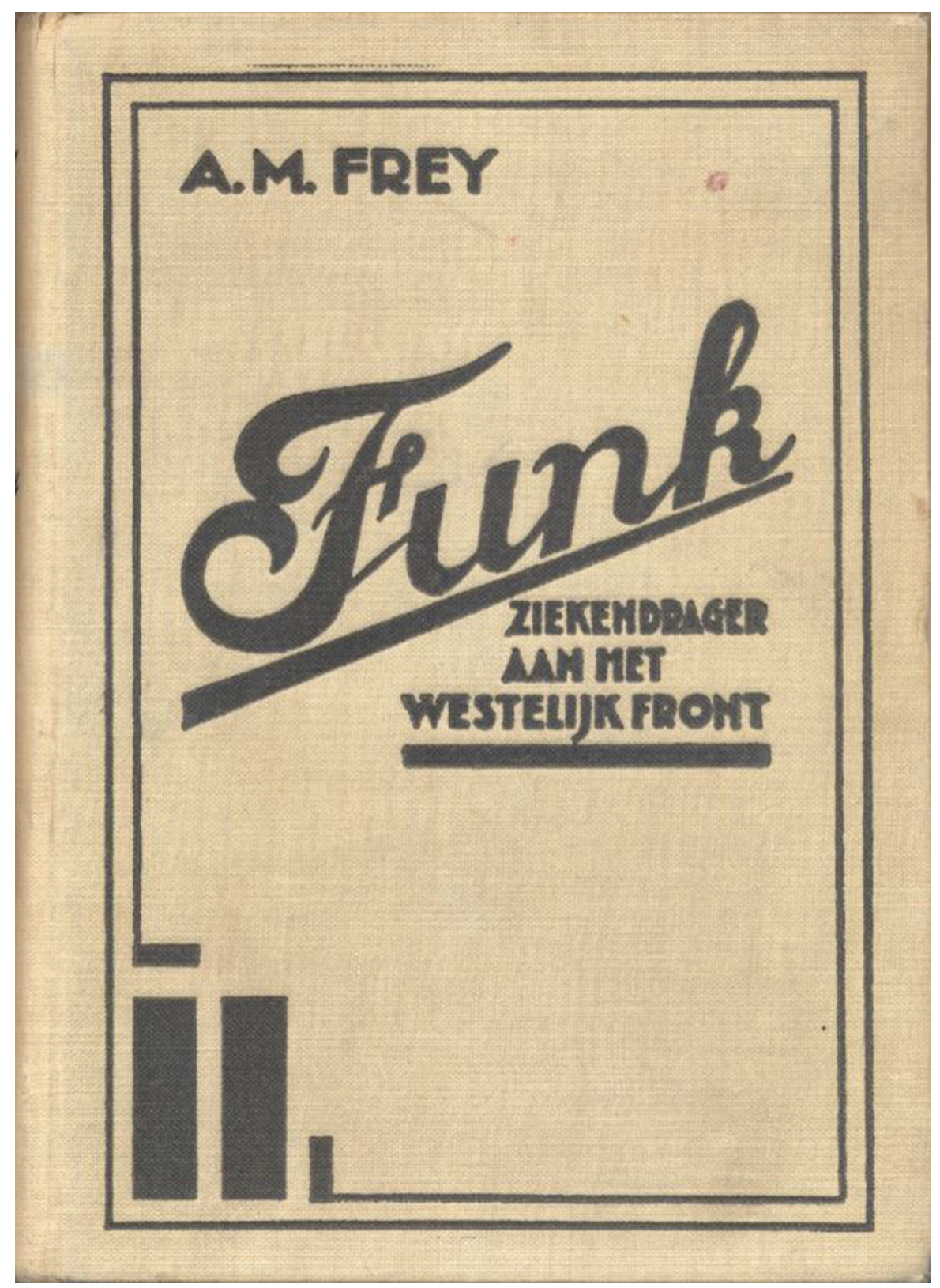

Abb.2:

rechten Ecke blickt ein Totenkopf aus einer Maske. Autorname und Buchtitel rahmen parallel zum Roten Kreuz den Bucheinband (Abb. 3).$^{10}$ Freys polnischer Übersetzung in der Reihe Książky o Wojnie (Bücher vom Krieg) waren - so die Verlagswerbung im Buch - u.a. die Romane von Erich Maria Remarque, Ernst Glaeser, Arnold Zweig, Jaroslav Hašek und Pierre Benoit vorausgegangen. Als „im Druck“ befindlich angekündigt wurden u.a. die Antikriegsromane von Joseph Roth, Henri Barbusse, John Dos Passos, Ernest Hemingway. Derart zu den großen Werken der Weltliteratur gezählt, war die polnische zugleich die letzte Übersetzung der Pflaster-

10 Die Abbildung dieses Umschlags, der auch bei dem ferngeliehenen Exemplar der Bibliotheka WSP Kielce fehlte, entstammt dem Kaufangebot des polnischen Antiquariats Archivum: https://archiwum.allegro.pl/oferta/apteczka-polowa-frey-okl-berman-i7450272567.htm [Zugriff: 30. Oktober 2020]. kästen. Eine französische Ausgabe gab es nicht, obwohl die Germanistin und Ärztin Cécile Knœrtzer(-Goguel) (18961978) im Juli 1929 eine gute Besprechung in der Revue Rhénane veröffentlicht und Romanpassagen unter dem Titel $L a$ Boîte aux Emplâtres übersetzt hatte (Knœrtzer 1929).

Hitler und sein ehemaliger Vorgesetzter, nun Gefolgsmann Max Amann boten dem als "ehemalige[n] Kamerad[en]" und ,reine[n] Arier"“ umworbenen Frey die Übernahme der Feuilletonredaktion des Völkischen Beobachters an, was Frey ebenso wie spätere Avancen ablehnte (HoffmannWalbeck 1984: 82f.; Greiner-Mai 1986: 250f.; Walter 1988: 20). ${ }^{11}$ Wenige Wochen nach der ,Machtergreifung' musste

11 Umworben noch nach Veröffentlichung der Pflasterkästen: Frey 1939? zit. nach Ernsting 2007: 71f. 


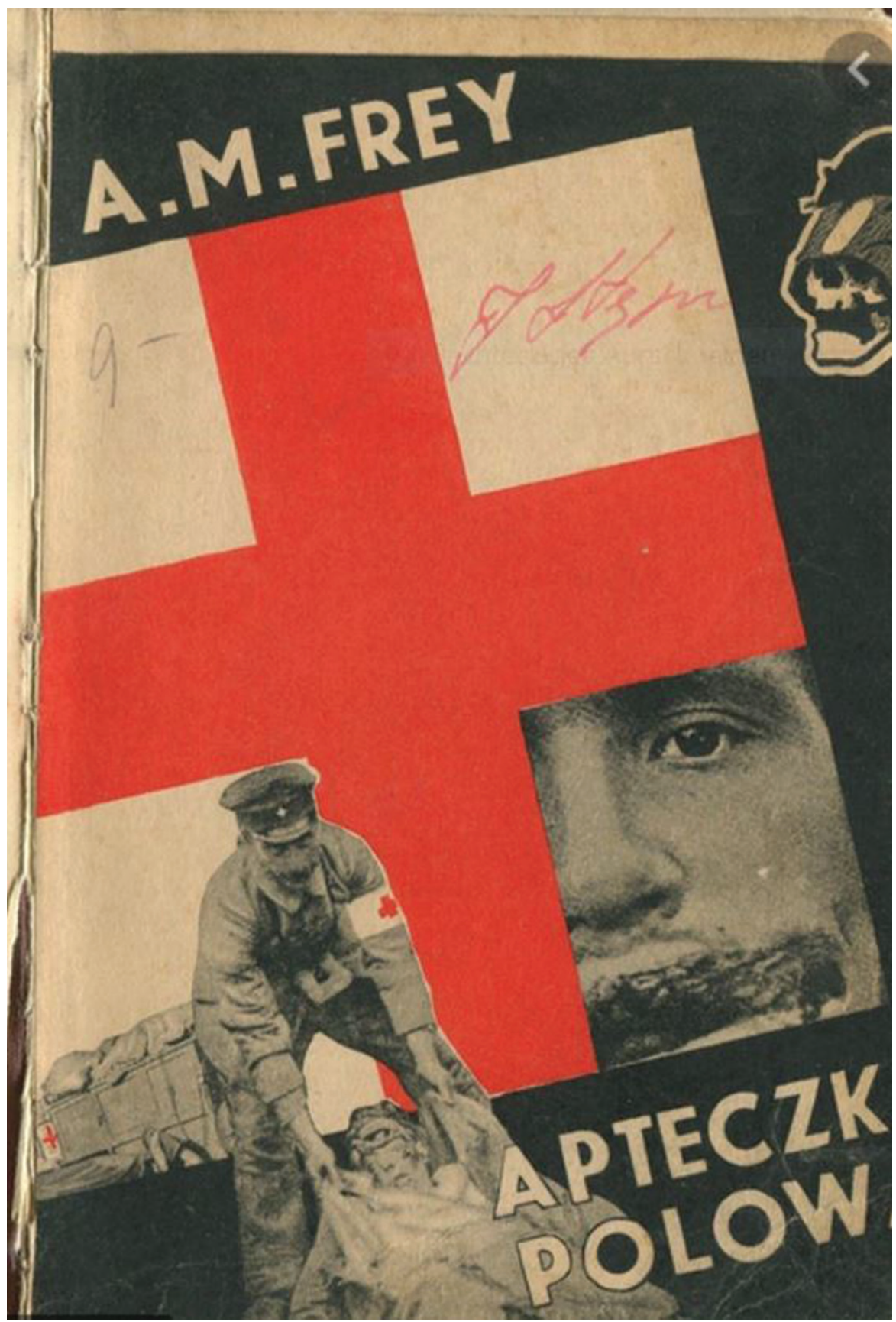

Abb.3:

Frey fliehen. Sein Antikriegsroman fiel den nationalsozialistischen Bücherverbrennungen zum Opfer, sein Name stand auf der Liste des schädlichen und unerwünschten Schrifttums (Treß 2003). ${ }^{12}$ Für Frey begann eine von Armut und Unsicherheit geprägte Exilzeit in Innsbruck und Salzburg, die sich nach dem ,Anschluss' Österreichs im März 1938 in Basel und später in Zürich fortsetzte. Er starb am 24. Januar 1957 in Zürich. ${ }^{13}$

12 Liste des schädlichen und unerwünschten Schrifttums, Stand vom 31. Dezembe 1938.

13 Abschiedsworte, gesprochen im Namen der Freunde von Dr. Walter Fabian am Montag, 28. Januar 1957, im Krematorium Zürich (Walter 1988: 257-267).
Nach dem Zweiten Weltkrieg war Frey den Deutschen ein Unbekannter. ${ }^{14}$ In den 1980er Jahren wurden Die Pflasterkästen im Zeichen des neuerwachten Forschungsinteresses an der zwischen 1933 und 1945 entstandenen „Exilliteratur" in den beiden Deutschland wiederentdeckt (Eckert 2020; Köpke 1989). Als 52. Band der Gustav-Kiepenheuer-Bücherei erschienen die Pflasterkästen 1984 in Leipzig und als Lizenzausgabe im Fischer Taschenbuch Verlag in Frankfurt am Main. Während der Leipziger Band die graphische schwarzweiß-Gestaltung der Gustav-Kiepenheuer-Reihe aufnahm, wurden die

14 In der Büchergilde Gutenberg, Frankfurt a.M. / Wien, erschienen in der Reihe "Bibliothek Exilliteratur" verschiedene Frey-Titel, u.a. Hölle und Himmel (1988). 
bundesrepublikanischen Pflasterkästen im Design der von Verlagslektor Ulrich Walberer betreuten und von dem Graphiker und Buchgestalter Max Bartholl gestalteten Reihe Verboten und verbrannt/Exil publiziert. Bei allen Büchern der Reihe nahm eine rote Banderole das untere Drittel des Umschlages ein; Autornamen, Titel, Verlag und Verlagssignet figurierten in schwarzer Schrift, während der Reihentitel in weißer Schrift hervorgehoben wurde. ${ }^{15}$ Zwei Drittel des Umschlages füllen körnige Schwarzweißfotografien. Die Vorderseite dominiert ein feldmäßig gerüsteter Sanitäter mit Rotkreuz-Armbinde auf einem morastigen Schlachtfeld, der sich zu einer Krankentrage hinunterbeugt. Als größtmöglichen Kontrast dazu, jedoch mit Freys Offizierskritik harmonierend, bietet die Rückseite die idyllische Kaffeetisch-Szene zweier Offiziere.

Man darf es eine Ironie des Schicksals nennen, dass ausgerechnet die 2007 einsetzende historische Forschung zu Hitlers ,frühen Jahren' seinen entschiedenen Gegner Frey der Vergessenheit entriss. ${ }^{16}$ Zuletzt spielte für Freys literarisches Nachleben auch die 100. Wiederkehr des Beginns des Ersten Weltkrieges eine Rolle, denn 2014 erschien die jüngste Ausgabe der Pflasterkästen in dem 2006 gegründeten Elsinor Verlag, der sich laut Eigenwerbung „zu Unrecht in Vergessenheit geraten[ener]" literarischer Werke annimmt - und so eben auch Freys Antikriegsroman wiederveröffentlichte. ${ }^{17}$

\section{ADRIENNE THOMAS UND IHR „ELSASS- LOTHRINGISCHER" ROMAN}

Das Manuskript des Bestsellers Die Katrin wird Soldat. Ein Roman aus Elsass-Lothringen hatten der Ullstein Verlag und ein halbes Dutzend weiterer Verlage zurückgewiesen (Metzger 1988: 141). Der Roman erschien 1930 im Propyläen Verlag Berlin, wurde in fünfzehn Sprachen übersetzt und zählte zu den meistverbreiteten pazifistischen Romanen der Weimarer Republik; schon im Erscheinungsjahr wurde er über 100.000 Mal verkauft. ${ }^{18}$ Geschrieben hatte inn die 33jährige Hertha Lesser geb. Strauch (1897-1980) unter ihrem nom de plume Adrienne Thomas; sie war in St. Avold geboren worden und zweisprachig in Metz aufgewachsen..$^{19} \mathrm{lhr}$ als Tagebuch eines jungen Mädchens abgefasster Antikriegsroman wurde seiner jugendlichen Hauptfigur und seines authentischen Tonfalls wegen auch als "Backfischliteratur" gelesen und wird heute zur Kinder- und Jugendbuchliteratur gezählt (Saletta 2017; Fuss 2001; Kinder- und Jugendliteratur 1999). Tatsächlich basiert Die Katrin wird Soldat auf dem inzwischen edierten Jungmädchentagebuch seiner Autorin aus der Zeit des Ersten Weltkrieges,

$15 \mathrm{Zu}$ der von Verlagslektor Ulrich Walberer betreuten Reihe „Verboten und verbrannt/ Exil“", die 1981 lanciert wurde und die 24 Titel umfasste, s. Fischer (2021), 1163; Lühe/Krohn (2005), 87-88. Zu Bartholl s. Dröge (o.J.), seit 2019 als Book on Demand zu haben.

162007 erschien eine Biographie Freys aus der Feder des Publizisten Stefan Ernsting 2010 publizierte der in Aberdeen lehrende Historiker Thomas Weber seine detailreiche Studie Hitler's First War (Plöckinger 2013).

17 "Gründung und Ausrichtung" https://www.elsinor.de/elsinor/ [Zugriff 10.07.2020]

18 Moens 1993; Keith-Smith 1997: 145-163. Der Roman trägt die Widmung „Dem Andenken von Arthur Lesser".

19 Dreidemy 2018; Puschak 2017; Orzechowski 2016, Rohlf 2005; Scholdt 2004; Bilsky 1995; Sinhuber1990; Metzger 1988. dessen Duktus sie bewusst beibehielt (Scholdt 2004). Die zweisprachige Romanheldin Catherine Lentz, jüngere Tochter aus großbürgerlich-jüdischem Hause in Metz wie ihre Autorin, lebt wie ihr geliebter Vater in Harmonie mit der französischen und deutschen Kultur. Zu ihrem 14. Geburtstag im Mai 1911 erhält sie ein Tagebuch, das sie umgehend mit Worten an eine ihr fehlende Freundin einweiht: „Viel erleben tu ich nicht, aber ich bin doch oft unzufrieden mit mir selbst und sehr unglücklich über die schrecklichen Zustände hier daheim." (Tagebucheintrag Metz, den 27. April 1911, Thomas 1987: 7). ${ }^{20}$ Das Tagebuch der lebenslustigen, hübschen und mit Gesangstalent begabten Catherine lässt seine Leser*innen an ihren spätpubertären Freuden und Nöten, Lieben und Launen in Familie und Schule teilhaben. Im Dezember 1913 verliebt sie sich in den Oberprimaner Lucien Quirin, Sohn eines deutschen Vaters und einer französischen Mutter, der sich im August 1914 mit seinen Klassenkameraden als Freiwilliger meldet. Die erst 17jährige Katrin lässt sich am 16. August als freiwillige Helferin beim Roten Kreuz am Metzer Bahnhof einstellen. Sie entwächst dem behüteten Herkunftsmilieu einer ,höheren Tochter' und geht in der Krankenpflege verwundeter Soldaten auf - wie Hertha Strauch selbst, die am 12. Mai 1915 in inr Tagebuch schrieb: „Ja, wenn ich auf der Bahn bin, unter all dem Elend, da fühle ich mich zuhause, eben weil ich lindern darf. Sonst aber nirgends. Überall überflüssig“" (Scholdt 2004: 29). Als „die Katrin“, zuletzt examinierte Hilfskrankenschwester in einem Krankenhaus in Plantières im damaligen „Stadtkreis Metz", am 9. Dezember 1916 ihr Tagebuch schließt, ist sie im Kriegskrankendienst erwachsen geworden, in dem sie Verwundung und Tod, auch den ihrer Liebe Lucien, ertragen lernte; sie wurde dabei jeder patriotischen Aufwallung und des für sie absurden, Verzweiflung und Tod säenden Krieges müde, auf dessen beiden feindlichen Seiten sie vertraute Freund*innen hatte. Sie stirbt kurze Zeit später an einer Lungenentzündung, wie die fiktive Freundin und Erbin des Tagebuchs Suzanne Lacy in einem „Nachwort“ berichtet.

Die Autorin der Katrin hingegen, die Tagebuchschreiberin Hertha Strauch, verabschiedete sich von ihrem „zweiten Ich“ schon am 7. Februar 1916, um nach der Schließung der unterfinanzierten Rote Kreuz-Station in Metz mit ihrer Familie nach Berlin zu ziehen und dort weiter in der Krankenpflege zu arbeiten. ${ }^{21}$ Nach Kriegsende absolvierte sie eine Gesangsund Schauspielausbildung und heiratete 1921 den Zahnarzt Arthur Lesser, der in jenem Jahr 1930 starb, in dem sie ihre Erinnerungen an den Ersten Weltkrieg unter dem Titel Die Katrin wird Soldat publizierte.

Während Catherine soldat : roman in der Übersetzung von Hélène Kra und Armand Pierhal mit einem Vorwort des Dichter-Diplomaten Jean Giraudoux (1882-1944) 1933 in drei Auflagen in Frankreich erschien, wurde das Originalwerk im nationalsozialistischen Deutschen Reich verbrannt und seine jüdische Autorin verfolgt. ${ }^{22}$ Adrienne Thomas floh nach

20 Den Romaninhalt fassen zusammen: Biener 2007, und Jung Seo 2003: 62-84.

21 Der letzte Tagebucheintrag erfolgt Metz, Montag den 7. Februar 1916 (Scholdt 2004: 206). 22 Die Übersetzerin Hélène Kra war Inhaberin einer deutschen Buchhandlung in Paris (Azuélos 2005: 175). Der französische Schriftsteller und Übersetzer Armand Pierhal (auch: Raphael Miranda) (1897-1976) übersetzte u.a. Ernst Robert Curtius, Konrad Heiden oder Ödon von Horvarth. Fischer (2021), 1072. 


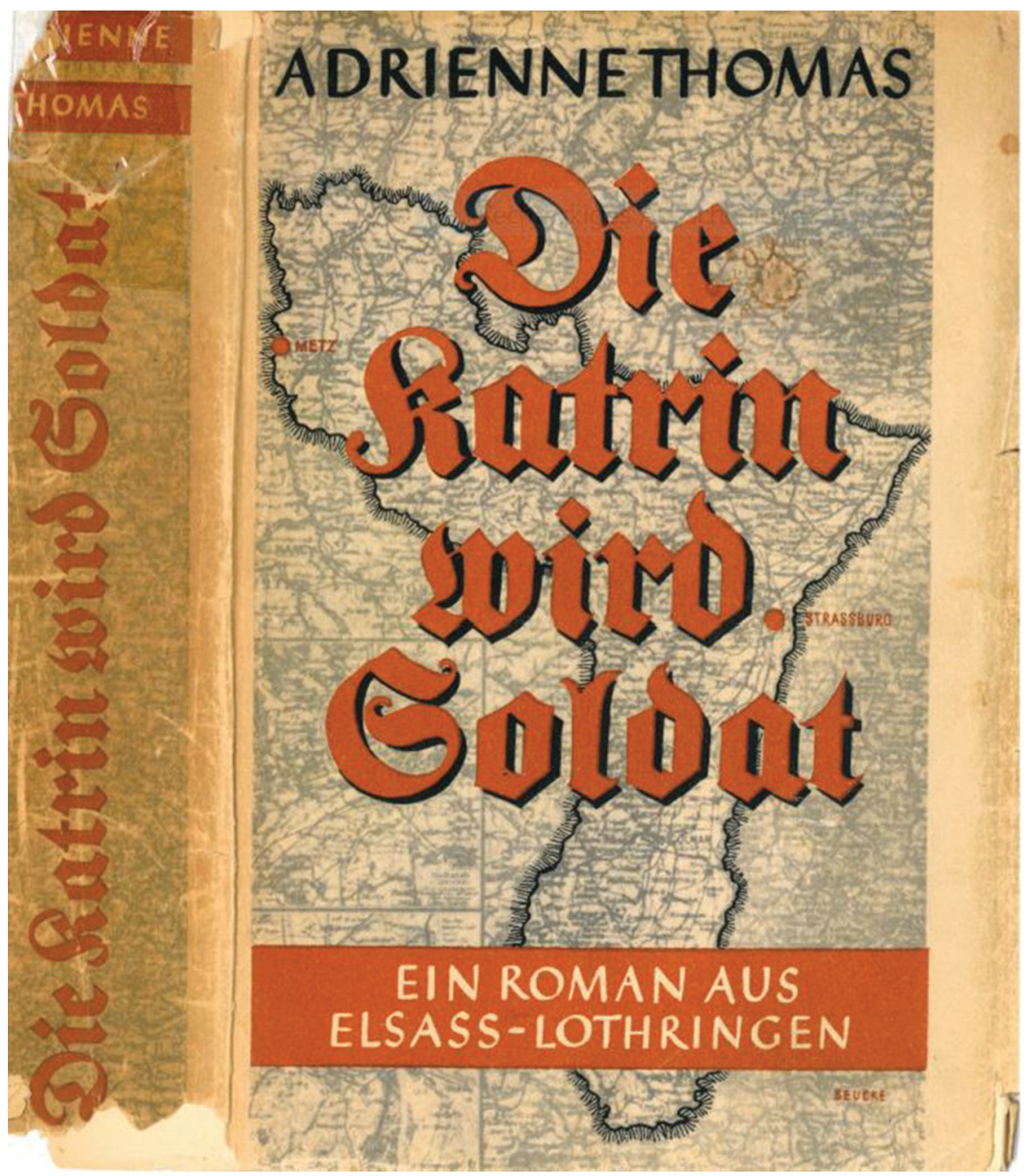

Abb.4:

Österreich, nach dessen ,Anschluss' nach Frankreich, wo sie 1940 im Frauenlager Gurs interniert wurde, abermals fliehen und in die USA entkommen konnte. ${ }^{23}$ Dort lernte sie ihren zweiten Ehemann, den österreichischen Sozialdemokraten, Spanienkämpfer und späteren österreichischen Verteidigungsminister Julius Deutsch (1884-1964) kennen, mit dem sie 1947 nach Österreich zurückkehrte. Als Schriftstellerin und international Vortragende geachtet, starb Adrienne Thomas am 7. November 1980 in Wien.

Wenden wir uns den Buchumschlägen und -einbänden zu, so stammt der cremefarbene Leineneinband der ersten deutschen Ausgabe von 1930 mit blauer und roter Prägung auf Vorderseite und Buchrücken (und rotem Seitenschnitt) von dem Graphiker und Bucheinbandgestalter Werner Beucke (19031940), der 1936 nationalsozialistische Aufträge annehmen sollte. ${ }^{24}$ Der Buchumschlag zeigt auf Vorderseite und Buchrücken die Landkarte des Reichslandes Elsass-Lothringen

23 S. Adrienne Thomas“ „Emigrationspfad“: Blumesberger, Susanne. Thomas Adrienne, geb. Hertha A. Strauch, verh. Deutsch, verh. Lesser, Ps. Erika Theobald, Erzählerin und Kinderbuchautorin: https://www.univie.ac.at/biografiA/daten/text/bio/thomas.htm [Zugriff: 30.06.2020].

24 http://www.germandesigners.net/designers/werner_beucke [Zugriff: 15.09.2020]. von 1905, über die in ziegelroter fetter Frakturschrift der Titel Die Katrin wird Soldat und auf die ein ziegelrotes Feld mit dem in (Trajan-)Majuskeln gehaltenen Untertitel Ein Roman aus Elsass-Lothringen gedruckt wurde. (Abb. 4) Rein graphisch gestaltet war hingegen der cremefarbene, in rot und grün mit Titel, Autorinnenname und Verlagskürzel bedruckte Bucheinband und -rücken der 1936 in der deutschsprachigen Abteilung des Exilverlages Allert de Lange in Amsterdam erschienenen Ausgabe der Katrin, die der über Paris in die Niederlande geflüchtete Graphiker Paul L. Urban (1901-1937) entworfen hatte. ${ }^{25}$ (Abb. 5) In den USA, Schweden und Dänemark erschienen 1931 ebenfalls Übersetzungen, teilweise von bedeutenden Übersetzer*innen wie Margaret Leland Goldsmith, 1940 wurde Katrien wordt soldaat in niederländischer Sprache publiziert.

1933 sorgte das Vorwort des Schriftstellers und Ministers für Propaganda der Regierung Daladier, Jean Giraudoux

25 Paul L. Urban, der vor seiner erzwungenen Flucht nach Paris von 1927 bis 1933 Grafiker in Berlin gewesen war, war für den Querido Verlag (Paris) und für den Allert de Lange Verlag in Amsterdam als Einbandgestalter tätig. Im Herbst 1936 emigrierte er in die Sowjetunion und nahm Quartier im Moskauer Hotel National. Es gibt von ihm nach dem Sommer 1937 keine Nachrichten mehr (Fischer 2021, 698; Löb 1989: 177-207). 


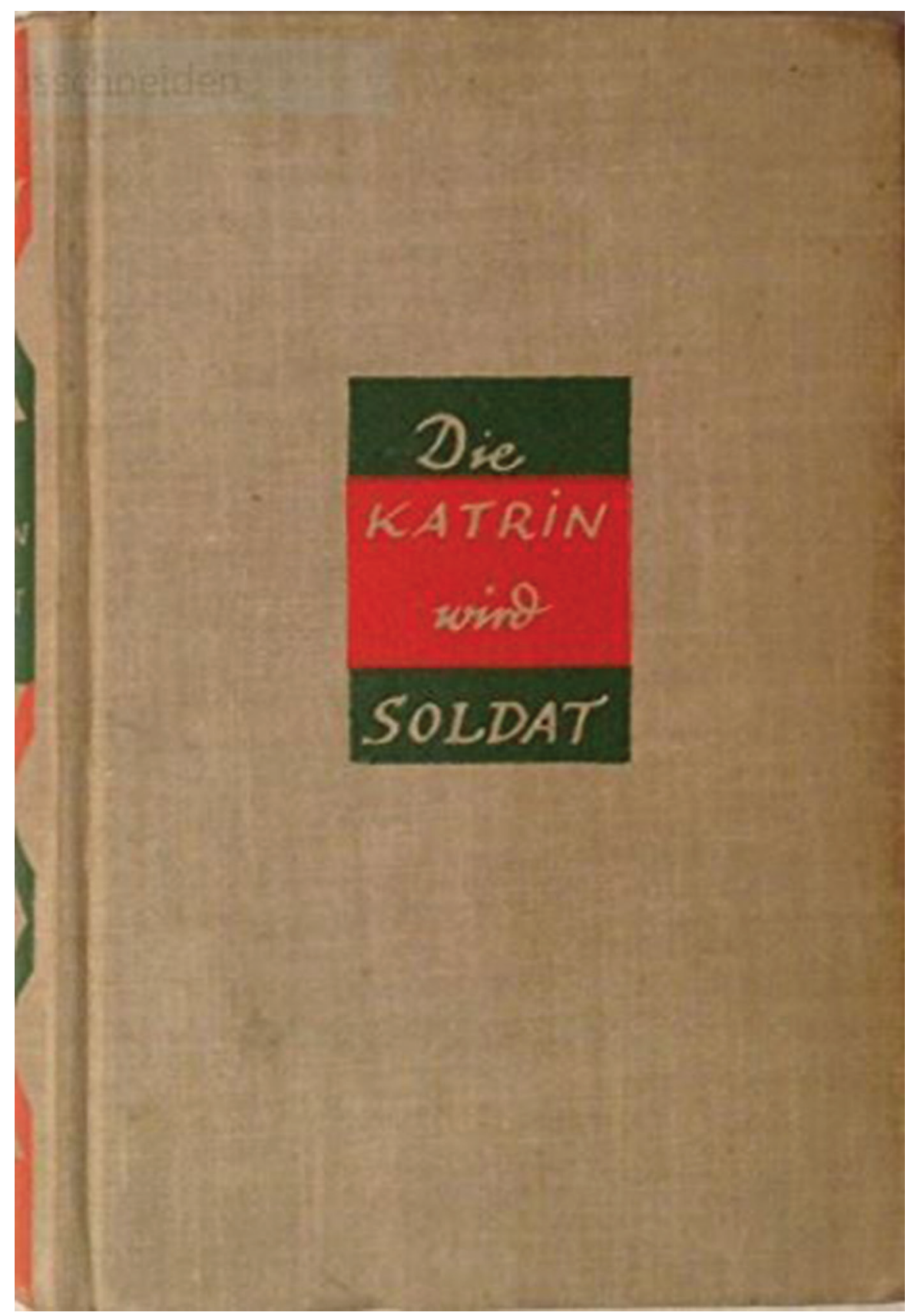

Abb.5:

(1882-1944), für reges Publikumsinteresse an der erwähnten französischen Übersetzung Catherine soldat; sie erschien in der Librairie Stock und trug auf der Vorderseite ein in ecru, beige und schwarz gehaltenes Aquarell von "Ergès“.

${ }^{26}$ Es zeigt eine vorne links ins Bild kommende jugendliche Rotkreuzschwester, hinter der sich ein den romantischen Stadtsilhouetten von Hansi ähnlicher Platz mit Brunnen öffnet; ein bewaffneter Soldat, Sanitäter und ein Sanitätswagen verweisen auf die Kriegszeiten. (Abb. 6) In der Zwischenkriegszeit, als die Erinnerung an den Ersten Weltkrieg

26 Zu Ergès konnte keine andere biographische Information gefunden werden als L'Héptameron: Contes de la Reine de Navarre in den Éditions Nilsson. frisch und die Zeit von 1871 bis 1918 in Lothringen besonders negativ konnotiert waren, erweckte Giraudoux' wohlwollendes Vorwort für das Roman aus Elsaß-Lothringen untertitelte Werk einer ,deutschen' Schriftstellerin deutliche Ablehnung in Frankreich (Teissier 1992; Gandebeuf 2009). Während die in Österreich lebende Adrienne Thomas dort nach dem Zweiten Weltkrieg verehrt wurde, musste sie in Deutschland und Frankreich erst wiederentdeckt werden. Fast gleichzeitig kamen Anfang der 1960er Jahre zwei Ausgaben der Katrin auf den westdeutschen Buchmarkt: 1961 erschien im mitgliederstarken Bertelsmann Buch-Club („Lesering“) Die Katrin wird Soldat als Lizenzausgabe in einem weißen Einband, auf dem Autorenname und Titel in alt(modisch) 


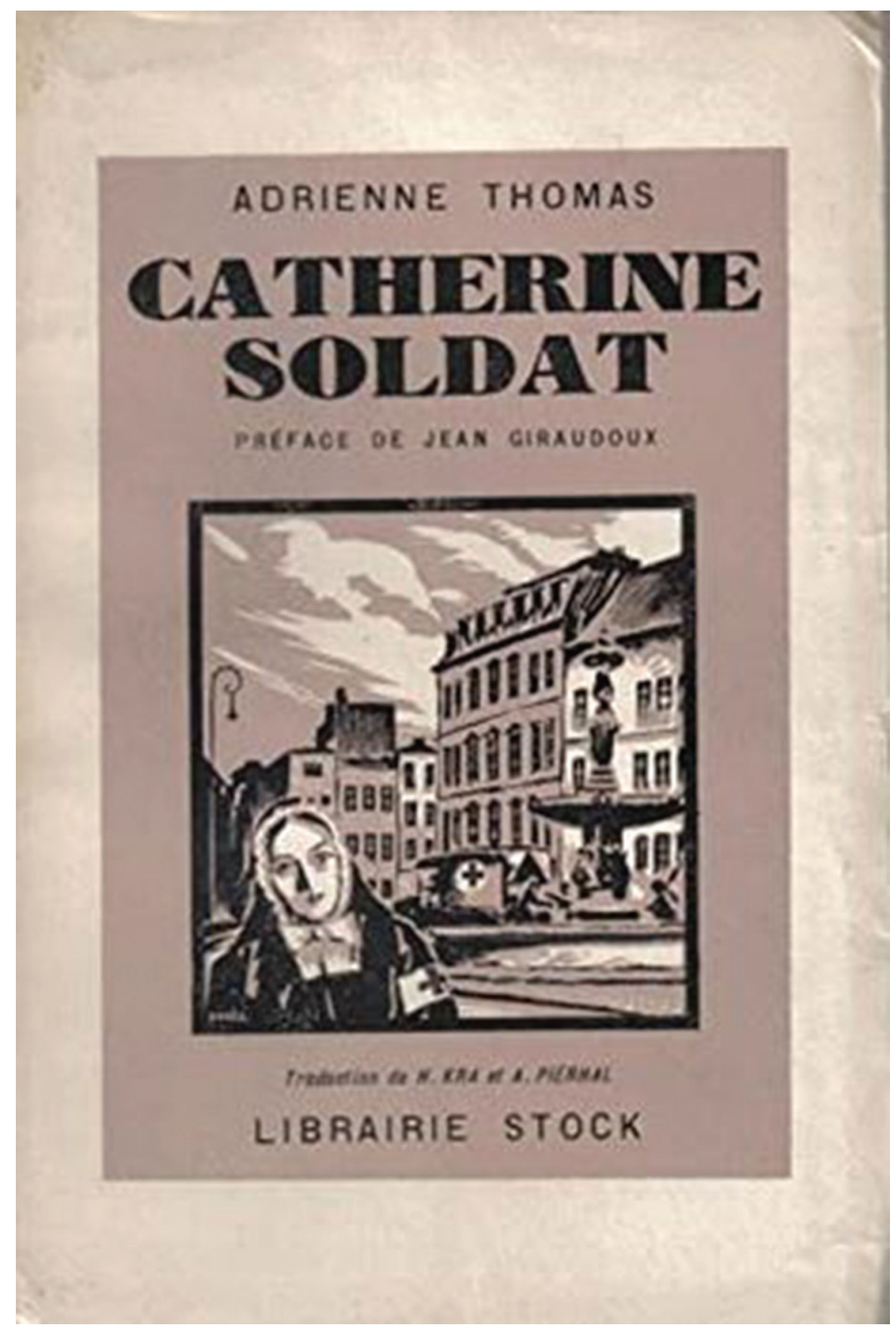

Abb.6:

er Schwabacher Schrift standen; wie diese in Grau und Rot gehalten waren auch die jugendliche, elegante Frauenfigur und die roten Kreuze. Der Publizist und langjährige Präsident der Israelitischen Kultusgemeinde München und Oberbayern Hans Lamm (1913-1985) ließ Adrienne Thomas' Katrin 1962 in seinem fünf Jahre zuvor in München gegründeten Ner Tamid-Verlag neu auflegen. ${ }^{27}$ Nicht betulich wie die Bertelsmann-Ausgabe, sondern vielmehr die Tragik der pazifistischen Erzählung betonend, ist auf dem orangefarbenen Schutzumschlag des moosgrünen Buches eine zur Faust

27 Ner Tamid bedeutet „Immerwährendes Licht“ (Sinn 2007: 122). geschlossene Hand zu sehen, die eine gebrochene Blume umkrampft; Autorenname und Titel stehen in der modernen amerikanischen Schreibschrift Brush Script. Ein Jahr nach Freys Pflasterkästen erschien auch Die Katrin wird Soldat in der Reihe Verboten und verbrannt/Exil des Fischer Taschenbuch Verlages im Jahr 1987. ${ }^{28}$

In Frankreich war Catherine soldat seit 1933 nicht mehr gedruckt worden. Dem langjährigen Präsidenten der Société d'Histoire du Pays Naborien, Denis Metzger (1937-2004),

28 Im gleichen Jahr 1987 wurde das Buch unter der Regie von Peter Deutsch $\left(^{*} 1939\right.$ verfilmt; im Goldmann Verlag erschien im Nachgang 1988 eine Neuauflage des Romans. 
gebührt das große Verdienst, sich nicht nur mit einer neuerlichen Publikation der Erstausgabe von Catherine soldat 1989 in den Éditions serpenoise, sondern auch in Vorträgen und Veröffentlichungen dafür eingesetzt zu haben, Adrienne Thomas in ihrem Heimatland Frankreich einer „ungerechten Vergessenheit" zu entreißen. ${ }^{29}$ Zwanzig Jahre später warb der Journalist und Leitartikler des Républicain Lorrain Jacques Gandebeuf (* 1926) mit einem reich bebilderten Band auch in Metz selbst für die Wiederentdeckung „[d'] une orpheline de Metz" (Gandebeuf 2009). Heute scheint Adrienne Thomas in Lothringen und Metz, das sie keineswegs liebte, weil es ,deutsch', sondern weil es ihre Heimat war, als „bedeutende Persönlichkeit des Humanismus und Pazifismus in Europa" wieder in das französische Kulturerbe aufgenommen. ${ }^{30}$ Seit 2004 verleiht die Stadtverwaltung von Saint-Avold jährlich während der Journées européennes du patrimoine einen nach Adrienne Thomas benannten Preis an junge Historiker*innen, die über die Geschichte ihrer Stadt arbeiten, und 2012 wurde eine der durch den Hauptbahnhof Metz führenden Passagen ihr zu Ehren passage Adrienne Thomas benannt.

Diese von der französischen Kulturpolitik lancierte ,europäische' Sichtweise auf Adrienne Thomas spiegelt sich in den Bucheinbänden sowohl in Frankreich als auch Deutschland und scheint damit ein „Einschwingen“ der französischen wie der deutschen Erinnerungskultur auf eine gemeinsame europäische Erinnerung an sie und wohl auch an den Ersten Weltkrieg zu demonstrieren, der als la Grande Guerre lange Jahre eine so viel bedeutendere Rolle in der französischen Erinnerungskultur spielt als in der deutschen. Die jüngste deutsche Ausgabe der Katrin ähnelt vom fotomontierten Einband her einer wenig später erschienenen französischen Publikation zu Adrienne Thomas. Beide Einbände zeigen in Sepiatönen

29 Thomas, Adrienne (1989), Catherine soldat : roman, trad. de l'allemand par Hélène Kra et Armand Pierhal; préface de Jean Giraudoux; avant-propos de Denis Metzger, Metz (Éditions Serpenoise). Zur Biographie von Denis Metzger s. Société d'Histoire du Pays Naborien http://www.shpn.fr/page2/page2.html und einen ausführlichen, auch Metzger Engagement für Adrienne Thomas würdigenden Nachruf von Charles Hiegel (2005). http:/ documents.irevues.inist.fr/bitstream/handle/2042/33976/ANM_2005_65.pdf?sequence=1 [Zugriff: 20.07.2020].

30 Zitat aus dem Absatz „Postérité“ des Artikels https://fr.wikipedia.org/wiki/Adrienne Thomas [Zugriff: 31.10 .2020$]$, der überwiegend basiert auf Jacques Gandebeuf und Les dossiers de la Société d'Histoire du Pays Naborien - 29 (Société d'Histoire du Pays Naborien, 28 rue des Américains, 57500 Saint-Avold) URL: http://www.shpn.fr/page76/ page76.html [Zugriff: 31.10.2020].

\section{Auswahlbibliographie:}

Frey, Alexander Moritz (1929), Die Pflasterkästen. Ein Feldsanitätsroman, Berlin, Gustav Kiepenheuer.

Frey (1919), Die Ernte. Mit drei Holzschnitten von Otto Nückel, Orchideengarten - Phantastische Blätter, hrsg. v. Karl Hans Strobl in Zusammenarbeit mit Alfred von Czibulka, Heft 3 (1919, 8-14). [https://digi.ub.uni-heidelberg.de/diglit/orchideengarten1919/0078, Zugriff: 27.07.2020].

Frey (1920), Spuk des Alltags. Elf Geschichten aus Traum und Trubel. Mit zwölf Holzschnitten von Otto Nückel, München, Delphin.

Frey (1929), Funk ziekendrager aan het Westelijk front (übersetzt von J. L. van der Moer), Utrecht, Universitas. das Porträtfoto der 16jährigen Hertha Strauch mit den in Die Katrin wird Soldat oft angesprochenen schwarzen Augen, dazu ihren Wirkungsort als junge Rotkreuz-Helferin Hertha/ Katrin, nämlich den Metzer Hauptbahnhof; seine markante Front samt eines deutschen Soldaten mit Pickelhaube bildet der deutsche Umschlag ab, einen Blick auf „Bahnsteig 4“ mit zivilen wie militärischen Wartenden gewährt der französische Bucheinband (Scholdt 2008). Auch die seit der Erfindung des Buchdrucks übliche, von Land zu Land differierende Einbandgestaltung bestätigt in ihrer Ähnlichkeit und zugleich in ihren spezifischen Unterschieden den deutsch-französischen Grundkonsens der Präsentation dieses nun beiderseitig neu wertgeschätzten pazifistischen Antikriegsromans.

Die künstlerische Auseinandersetzung mit dem Ersten Weltkrieg geschah und geschieht an den beiden pazifistischen Antikriegsromanen von Alexander Moritz Frey und Adrienne Thomas literarisch. Selten wird dabei die sich verändernde graphisch-buchgestalterische Präsentation der Werke beachtet, doch ist sie von eigener Aussagekraft. Georg Salter, der von den Nationalsozialisten zur Emigration gezwungene bedeutende künstlerische ,Gebrauchsgraphiker", soll über das „heroische Dasein“ eines Schutzumschlages gescherzt haben, dass dieser „auf eine Tat hin[ziele], die zugleich die Erfüllung des Lebenszweckes und Untergang für inn bedeute. Diese Tat sei [...] die Herbeiführung des Kaufentschlusses. Komme es dazu, so sei der Zweck des Schutzumschlages erfüllt, er überlebe das erste Lesen des Buches selten“ (Schauer 2003: 130f.). Tatsächlich sind die Schutzumschläge der beiden Antikriegsromane von Adrienne Thomas und Alexander Moritz Frey heute meist verloren, doch die Bucheinbände der Erstausgaben und der Übersetzungen, der Auflagen in Exilverlagen und der Nachkriegsauflagen berichten in der Art von Jahresringen eines Baumes von der wechselvollen Rezeptionsgeschichte der Romane Die Pflasterkästen und Die Katrin wird Soldat - Freys Roman harrt in Frankreich noch der Entdeckung, Thomas' Roman scheint gerade durch seine von Frankreich aus lancierte neue ,europäische' Rezeption zur Heilung der Wunden beitragen zu sollen, die der Große, der Erste Weltkrieg vor mehr als einem Jahrhundert geschlagen hat.

Frey (1931), Apteczka polowa: powieść (übersetzt von Marceli Tarnowski [1899-1944]), Warsawa (Towarzystwo Wydawnicze «Rój »).

Frey (1986 [1929]), Die Pflasterkästen. Ein Feldsanitätsroman. Mit einem Nachwort von Herbert Greiner-Mai, Frankfurt a.M, Fischer Taschenbuch.

Thomas, Adrienne (1930), Die Katrin wird Soldat. Ein Roman aus Elsass-Lothringen, Berlin, Propyläen, (116.-125. Tsd. 1932).

Thomas (1931), Cathérine Lentz' kärlek [Catherine Lentz' Liebe]. Roman från Elsass-Lothringen, Stockholm (Aktiebolaget Skoglunds Bokförlag], übers. V. Karl Fägersten. 
Thomas (1931), Cathérine's dagbog: Roman fra Elsass-Lothringen Oversat fra tysk af Peter Sennet, Købnhavn, Wilhelm Hansen.

Thomas (1931), Katrin becomes a soldier. Translated by Margaret $\mathrm{L}$. Goldsmith, Boston, Little, Brown \& Cie.

Thomas (1936), Die Katrin wird Soldat: ein Roman aus Elsaß-Lothringen, 125. Tsd. Amsterdam, Allert de Lange.

Thomas (1940), Katrien wordt soldaat : een roman uit Elzas-Lotharingen. Geautoriseerde vertaling door J. G. Lobel, Utrecht, Bijleveld.

Thomas (1987), Die Katrin wird Soldat (Verboten und verbrannt/Exil), Frankfurt a.M., Fischer.

Atze, Marcel (2006), „Alexander Moritz Frey“ in Asendorf, Manfred / von Bockel, Rolf (Hg.): Demokratische Wege. Ein biographisches Lexikon. Stuttgart, J. B. Metzler, $187 \mathrm{ff}$.

Azuélos, Daniel (2005), „Deutsche Buchhandlungen“, in Colin, Nicole / Defrance, Corine / Pfeil, Ulrich / Umlauf, Joachim (Hg.), Lexikon der deutsch-französischen Kulturbeziehungen, 2. überarb. und erw. Aufl., Tübingen, Narr Francke Attempto, 174-178.

Biener, Rebecca (2007), Die literarische Verteidigung des kleinen Glücks am Beispiel der Autorin Adrienne Thomas, Dissertation Uni Siegen von 2005 [https://dspace.ub.uni-siegen.de/bitstream/ ubsi/286/1/biener.pdf, Zugriff: 02.07.2020].

Bilsky, Lisa A. (1995), Adrienne Thomas, Getrud Isolani, and Gabriele Tergit. German-Jewish women writers and the experience of exile, Madison, $\mathrm{U}$ [niversity of Wisconsin.

Chrambach, Eva (2005), „Salter, Georg“, in Neue Deutsche Biographie 22, 397-398 [https://www.deutsche-biographie.de/ pnd123337836.html\#ndbcontent, Zugriff: 02.07.2020].

Dorén, Peter Nils (2003), "Zwischen Klassik und Moderne. Zur Typographie von Georg Salter in seinen Berliner Jahren", in Holstein, Jürgen (Hg.), Georg Salter. Bucheinbände und Schutzumschläge aus Berliner Zeit 1922-1934, Berlin, Holstein, 140-147.

Dreidemy, Lucile (2018), „Adrienne Thomas“, in Leclerc, Hélène (dir.), Le Sud-Ouest de la France et les Pyrénées dans la mémoire des pays de langue allemande au XXe siècle, Toulouse, Le Pérégrinateur éditeur, $187 \mathrm{f}$.

Dröge, Kurt (o. J.), Die Neue Arche Bücherei und Max Bartholl. Zum Wandel des Buchdesigns kleiner Reihen, Book on Demand.

Eckert, Brita (2020), Die Anfänge der Exilforschung in der Bundesrepublik Deutschland bis 1975. Ein Überblick [https://literaturkritik. de/public/Eckert-Exilforschung.pdf, Zugriff: 15.09.2020].

Ernsting, Stefan (2007), Der phantastische Rebell Alexander Moritz Frey oder Hitler schießt dramatisch in die Luft, Zürich, Atrium.

Fischer, Ernst (2021), Geschichte des deutschen Buchhandels im 19. und 20. Jahrhundert, Bd. 3: Drittes Reich und Exil, Teil 3: Der Buchhandel im deutschsprachigen Exil 1933-1945, Teilband 1, Berlin/Boston: Walter de Gruyter.

Fuss Phillips, Zlata (2001), German Children's Literature in Exile 1933-1950: Biographies and Bibliographies, München, de Gruyter Saur.

Gandebeuf, Jacques (2009), Adrienne Thomas le Fantôme Oublié de la Gare de Metz, Metz, Serpenoise.

German Literature and the First World War: The Anti-War Tradition. Collected essays by Brian Murdoch (2017), London, New York, Routlegde.
Greiner-Mai, Herbert (1984), „Nachwort: Auf der Suche nach Alexander Moritz Frey“, in Alexander Moritz Frey, Die Pflasterkästen, Frankfurt a.M., Fischer, S. 247-254.

Hansen, Thomas S. (2005), Classic Book Jackets. The Design Legacy of George Salter. Foreword by Milton Glaser, New York, Princeton Architectural Press.

Hoffmann-Walbeck, Katrin (1984), Alexander M. Frey. (Allegorische) Phantastik und Groteske als Mittel zur Zeitkritik, Frankfurt a.M., Peter Lang.

Jung Seo, Yun (2003), Frauendarstellungen bei Adrienne Thomas und Lili Körber, Marburg, Tectum.

Keith-Smith, Brian (Hg.) (1997), German Woman Writers 1900-1913. Twelve Essays, Lewiston/New York, Mellen.

Kiepenheuer, Noa (Hg.) (1951), Vierzig Jahre Kiepenheuer 19101950. Ein Almanach, Weimar, Gustav Kiepenheuer.

Kinder- und Jugendliteratur im Exil 1933-1950. Mit einem Anhang Jüdische Kinder- und Jugendliteratur 1933-1938. Eine Ausstellung der Sammlung Exil-Literatur der Deutschen Bücherei Leipzig (1999), Leipzig, Deutsche Bibliothek.

Knœrtzer, Cécile (1929), „La Guerre vécue et décrite par un infirmier. "La Boîte aux Emplâtres » de A. M. Frey“, in La Revue Rhénane, 9. Jg. Heft 10 (Juli), 42-45.

Köpke, Wolf / Winkler, Michael (Hg.) (1989), Exilliteratur 1933-1945, Darmstadt, Wissenschaftliche Buchgesellschaft.

Löb, Kurt (1989), 'Drei deutschsprachige graphische Gestalter als Emigranten in Holland (1925-50)', Philobiblon, 177-207.

Lühe, Irmela von der / Krohn, Claus-Dieter (Hg.) (2005), Fremdes Heimatland. Remigration und literarisches Leben nach 1945, Göttingen, Wallstein.

Metzger, Denis (1988), Les origines et les attaches mosellanes d'Adrienne Thomas (1887-1980). Une romancière de langue allemande et témoin de son temps, [http://documents.irevues.inist. fr/bitstream/handle/2042/34452/ANM_1987_137.pdf?seq, Zugriff: 02.07.2020].

Moens, Herman (1993), „Die Katrin wird Soldat. A fictionalized Diary of the First World War" in Keith-Smith, Brian, German women writers 1900-1933, Lewiston, Mellen, 145-163.

Orzechowski, Simone (2016), „Catherine part en guerre („Die Katrin wird Soldat") d'Adrienne Thomas. Fiction et réalité de la vie d'une femme en 1914-1918“, in Gemeaux, Christine de (dir.), Genre, Femmes et Guerre: perspectives européennes sur la Grande Guerre, Tours, Université François Rabelais.

Plöckinger, Othmar (2013), Unter Soldaten und Agitatoren. Hitlers prägende Jahre im deutschen Militär 1918-1920, Paderborn, Schöningh.

Puschak, Christiana (2017), Bekenntnis zur Verantwortung des Künstlers. Adrienne Thomas' autobiographisches Schreiben. Zwischenwelt. 2017, Nr. 4 Dezember 2017. Lebensspuren. Autobiographik von Exil, Widerstand, Verfolgung, 37-39.

Rohlf, Sabine (2005), „Zuhause war ich nur noch an irgend einem Schreibtisch": Autobiographie, Exil und Autorschaft in Texten von Irmgard Keun und Adrienne Thomas", in Exilforschung - Internationales Jahrbuch, 23, 128-149.

Saletta, Ester (2017), "Gendermotive in Adrienne Thomas' Mädchenromanen der 1930er Jahre: Erfahrungsgeschichten 
einer Frauenemanzipation im Krieg“, in Blumesberger, Susanne / Thunecke, Jörg (Hg.), Deutschsprachige Kinder- und Jugendliteratur während der Zwischenkriegszeit und im Exil, Frankfurt a.M., Peter Lang, 217-230.

Sas, Britt (2011), „La couverture, un objet culturel“, in L'Atelier des icônes. Carnet de recherches [http://histoirevisuelle.fr/cv/icones/1818, Zugriff: 15.09.2020].

Schauer, Georg Kurt (2003), „Georg Salter. Ein Rückblick“, in Holstein, Jürgen (Hg.), Georg Salter. Bucheinbände und Schutzumschläge aus Berliner Zeit 1922-1934, Berlin, Holstein, 129-132.

Scholdt, Günter (2008), Adrienne Thomas: Die Katrin wird Soldat und Anderes aus Lothringen (= Sammlung Bücherturm Bd. 9). Mit einem Nachwort von Günter Scholdt, St. Ingbert, Röhrig.

Scholdt, Günter (2004), „Nachwort“, in ders. (Hg.), Adrienne Thomas. Aufzeichnungen aus dem Ersten Weltkrieg. Ein Tagebuch (= Selbstzeugnisse der Neuzeit Bd. 14), Köln u.a., Böhlau, 207-224.

Sinhuber, Katrin (1990), Adrienne Thomas. Eine Monographie, phil. Diss. Wien.

Sinn, Andrea (2008), „Und ich lebe wieder an der Isar". Exil und Rückkehr des Münchner Juden Hans Lamm. Mit einem Geleitwort von Charlotte Knobloch, München, R. Oldenbourg.
Teissier, Gerard (1992), Jean Giraudoux et Catherine soldat. Cahiers Jean Giraudoux 21 (Le vingt-et-unième volume des Cahiers de l'écrivain et dramaturge français) Figures juives chez Jean Giraudoux, Éditions Grasette \& Fasquelle.

Thema - Stil - Gestalt. 1917-1932. 15 Jahre Literatur und Kunst im Spiegel eines Verlages (1984). Katalog zur Ausstellung anlässlich des 75jährigen Bestehens des Gustav Kiepenheuer Verlages, Leipzig/Weimar, Gustav Kiepenheuer.

Trebesch, Jochen (2007), Jean Giraudoux 1882-1944 (= Diener zweier Herren - Diplomaten-Autoren des 20. Jahrhunderts), Berlin, Nora.

Treß, Werner (2003), ,Wider den undeutschen Geist‘. Bücherverbrennung 1933, Berlin, Parthas

Treß, Werner (Hg.) (2009), Verbrannte Bücher 1933. Mit Feuer gegen die Freiheit des Geistes, Bonn, Bundeszentrale für politische Bildung, 234-241.

Walter, Hans-Albert (1988), Der Meisterzeichner von Nachtstücken. Alexander Moritz Frey - wiederzuentdecken, Frankfurt a.M., Büchergilde Gutenberg.

Weber, Thomas (2011), Hitlers Erster Krieg: Der Gefreite Hitler im Ersten Weltkrieg - Mythos und Wahrheit, Berlin, Propyläen. 